du jeune homme à celles des 2 parents : l'analyse s'avéra assez convaincante aux yeux des autorités d'immigration pour qu'elles accordent le permis de séjour. Conclusion des auteurs : leur méthode permet d'établir une relation de paternité, parfois même "in absentia ".

Un article de Lancet [2] paru en décembre 85 , s'attaque au problème des jumeaux, mono ou dizygotes. La question est simple si les nouveau-nés sont de sexe différent ou si, au contraire, il n'existe qu'un sac amniotique. Mais d'après l'analyse de la littérature faite par les auteurs, dans $50 \%$ des cas, les jumeaux sont de même sexe, l'amnios et le chorion sont doubles et l'on ne peut conclure. Les tests employés jusqu'à présent sont multiples, complexes et laissent une marge d'erreur non négligeable. Au contraire, les empreintes d'ADN devraient donner une réponse formelle : les jumeaux monozygotes ont des bandes identiques; s'ils sont dizygotes, il n'y a qu'une chance sur $10^{8}$ pour qu'il en soit ainsi. La méthode a été appliquée à 12 paires de jumeaux, la validité étant immédiatement testable sur 7 d'entre elles (2 placentas monochorioniques et 5 paires de sexes différents). Elle a été aussi employée sur des triplés nés de deux femmes traitées par des médicaments inducteurs de fertilité; dans les deux cas les triplés étaient tous différents.

Un dernier article de médecine légale explore la possibilité d'identifier des taches de sang et de sperme [3]. Les auteurs ont pu extraire de l'ADN non dégradé (indispensable pour la validité de la méthode) de taches datant de plusieurs mois, et même de quelques années, et parvenir à une identification avec les mêmes sensibilité et précision que sur les fluides frais. Il est également possible, après un viol remontant à quelques heures, d'en identifier le responsable à partir d'un prélèvement vaginal. Dans ce cas il faut d'abord éliminer l'ADN de la femme, majoritaire, par lyse préférentielle, les spermatozoïdes étant plus résistants à la lyse par certains détergents. La sensibilité de la technique est devenue telle qu'il suffit de 5 microlitres de sperme ou de 60 microlitres de sang pour réaliser une analyse complète.

En quelques semaines donc, une moisson exceptionnelle de résultats a été engrangée. Les méthodes seront bientôt utilisables pratiquement en médecine légale et devraient, selon les auteurs, révolutionner celle-ci. Il faudra toutef ois les confronter dans un premier temps, aux techniques actuellement en usage ainsi qu'à d'autres méthodes utilisant l'ADN mais qui paraissent moins sensibles et moins générales. Les sondes feront probablement l'objet de brevets et devraient être disponibles d'ici quelques mois, la commercialisation passant par le "Lister Institute".

J.C.D.

I. Jeffrcys A J, Brookficld JFW, Scmeonoff R Positive identification of an immigration test-case using human DNA fingerprints. Nature 1985; 317: 808-9.

2. Hill AVS, Jeffrcys AJ. Usc of minisatellitc DNA probes for determination of twin zygosity at birth. Lancet 1985; ii: 1394-5.

3. Gill P, Jeffreys AJ, Werrett DJ. Forensic application of DNA "fingerprints". Nature 1985; 318: $577-9$.

\title{
Epuration enzymatique de la bilirubine sanguine
}

Les traitements courants de l'ictère sévère du nouveau-né sont la photothérapie et surtout l'exsanguinotransfusion. Quoique très efficace, cette dernière n'est pas dépourvue d'inconvénients, en particulier liés aux craintes qu'inspirent actuellement les contaminations virales.

Lavin et coll. (Boston) [I] ont eu l'idée d'éliminer spécifiquement la bilirubine sanguine par une méthode enzymatique. Ils ont utilisé une préparation de bilirubine oxydase extraite de Myrothecium verrucaria, qui transforme la bilirubine en biliverdine puis en d'autres produits d'oxydation mal identifiés. Ils ont testé l'innocuité de ces composés aux points de vue tératogène, mutagène et cytotoxique.

La technique est relativement simple : l'enzyme est immobilisée par fixation covalente sur des billes d'agarose et possède dans ces conditions une demivie d'activité de 60 heures. Elle est insérée dans une colonne en verre reliée à une pompe péristaltique et maintenue à une température physiologique; la colonne est lavée avant usage par une solution isotonique stérile contenant de l'héparine. Ce réacteur a été testé jusqu'à présent sur des solutions de bilirubine liée à de la sérum albumine humaine, sur du sang humain in vitro et, in vivo, sur des rats Gunn. Rappelons que les rats Gunn présentent un déficit en glucuronyltransférase et ont un taux de bilirubine plasmatique oscillant autour de $300 \mu \mathrm{M}$ (environ I75 mg par litre). On sait par ailleurs que le seuil de bilirubinémie exigeant une exsanguinotransfusion est généralement fixé à $200 \mathrm{mg}$ par litre chez le nouveau-né à terme et plus bas chez le prématuré. Les résultats paraissent probants : in vitro, un passage unique sur la colonne fait disparaitre $90 \%$ de la bilirubine, le plasma passant du jaune au vert foncé. Chez les rats Gunn, le taux de bilirubine diminue de moitié en 30 minutes; l'équilibre ionique et le niveau des principaux métabolites sont très peu modifiés; la seule perturbation est une hémolyse modérée, que des améliorations techniques devraient maîtriser, notamment en ajoutant de l'héparinase immobilisée au réacteur. Cette technique d'épuration extracorporelle très sélective et relativement peu encombrante présente des avantages sur l'exsanguinotransfusion et les autres méthodes d'adsorption. Les auteurs espèrent qu'elle pourra bientôt être appliquée avec succès au traitement de l'ictère sévère du nouveauné. Ils pensent en outre que son emploi aidera à généraliser le concept des réacteurs à enzyme, susceptibles de débarrasser spécifiquement le sang de malades de substances toxiques endogènes ou exogènes.

J.-C. D.

I. Lavin A, Sung C, Klibanov AM, Langer R. Enzymatic removal of bilirubin from blood: a potential treatment for neonatal jaundice. Science 1985; 230: 543-5. 